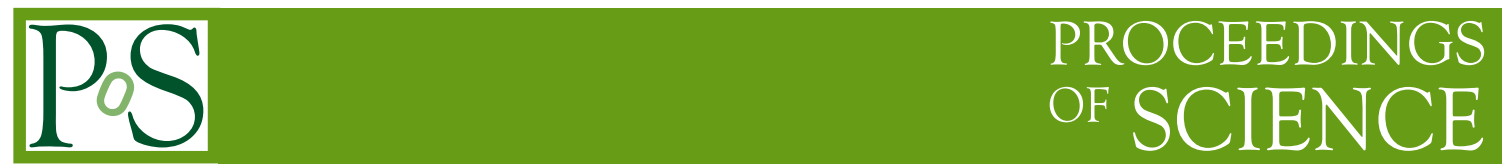

\title{
Unusual PMT behaviour in KamLAND
}

\author{
Alexandre Kozlov* \\ Research Center for Neutrino Science, Tohoku University, Japan \\ E-mail: kozlov@awa.tohoku.ac.jp
}

The origin of a high dark-pulse rate from the 1879 KamLAND 17-inch and 20-inch inner detector photo-multipliers is discussed. The observed phenomenon may play an important role in the proposed next generation of large scintillator detectors such as LENA in Europe and HANOHANO in the United States.

International workshop on new photon-detectors PD07

June 27-29 2007

Kobe University, Kobe, Japan

* Speaker. 


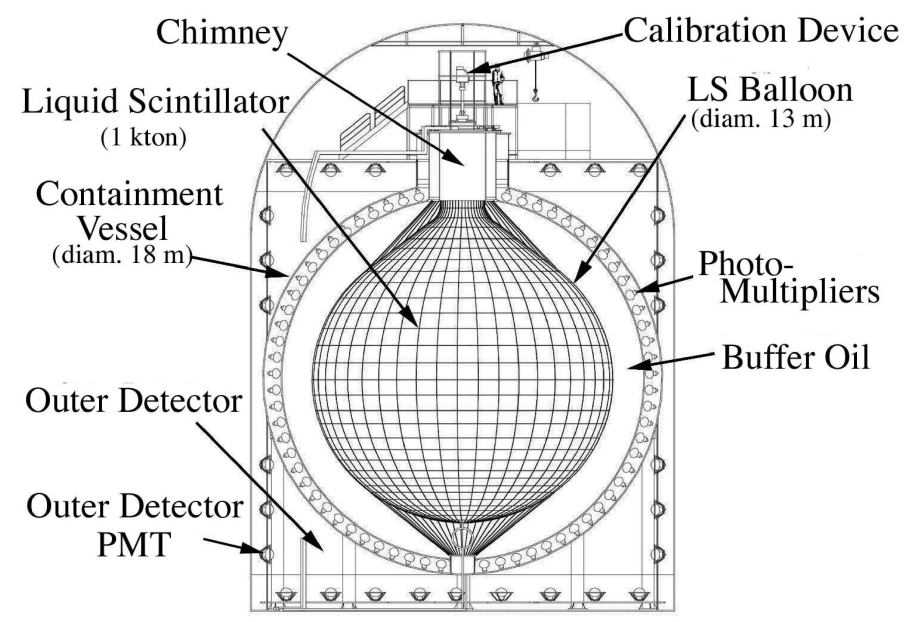

Figure 1: Schematic diagram of the KamLAND detector.

Kamioka Liquid scintillator Anti-Neutrino Detector (KamLAND), situated in the underground laboratory 'Kamioka mine' in the mountainous region of western Japan occupies the rock cavity previously used by the Kamioka nucleon decay experiment (Kamiokande) [1]]. The main goal of the KamLAND experiment [2] is to investigate the oscillation of $\bar{v}_{e}$ 's emitted from distant nuclear reactors. A combination of large detector mass, high $\bar{v}_{e}$ flux from Japanese nuclear reactors (located at averaged distance of $\sim 180 \mathrm{~km}$ ), and an ultra low background level allows us to investigate the oscillation solution of the so-called 'solar neutrino problem' [2] and achieve a precise measurement of $\Delta m^{2}$ [级. The KamLAND detector (Figure 1) consists of a 1 kton ultra-pure Liquid Scintillator (LS) enclosed in a 13-m-diameter spherical balloon made from 135 - $\mu \mathrm{m}$-thick transparent nylon/EVOH composite film. The balloon is supported and fixed by a network of kevlar ropes. The LS is composed of $80 \%$ dodecane, $20 \%$ pseudocumene (1,2,4-Trimethylbenzene), and $1.36 \pm 0.03 \mathrm{~g} / \mathrm{l}$ of PPO (2,5-Diphenyloxazole) as a fluor. A buffer of dodecane and isoparaffin oils between the balloon and an 18-m-diameter spherical stainless-steel containment vessel supports the balloon and provides a shield from external $\gamma$-rays. A 34\% photo-cathode coverage of an Inner Detector (ID) is achieved by 1,879 photomultiplier tubes (PMTs) mounted onto the inner surface of the containment vessel. Most of the KamLAND ID PMTs (1,325) are fast PMTs with a 17inch-diameter photocathode (R 7250 type), developed for KamLAND by Hamamatsu Photonics in cooperation with Tohoku University. The rest of the PMTs (554) are represented by older 20-inch Hamamatsu Photonics PMTs (R 1449 type) which were initially designed and produced for the Kamiokande experiment [ $₫$. The containment vessel is surrounded by a $3.2 \mathrm{kton}$ water-Cherenkov muon veto detector, the so-called Outer Detector (OD), equipped with 225 of the Hamamatsu Photonics 20-inch PMTs.

Properties of the newly developed 17-inch PMTs were studied extensively during the KamLAND construction period. In 1999 the stability of 12 PMTs was tested during a 1000 hour darkpulse rate measurement. During the test the dark-pulse rate remained at $5-7 \mathrm{kHz}$ at the discriminator level of $1 / 4$ of photo-electron equivalent (p.e.). The results of the test for two of the PMTs are shown in Figure 2 . The test was performed in a room with no temperature control from the begin- 

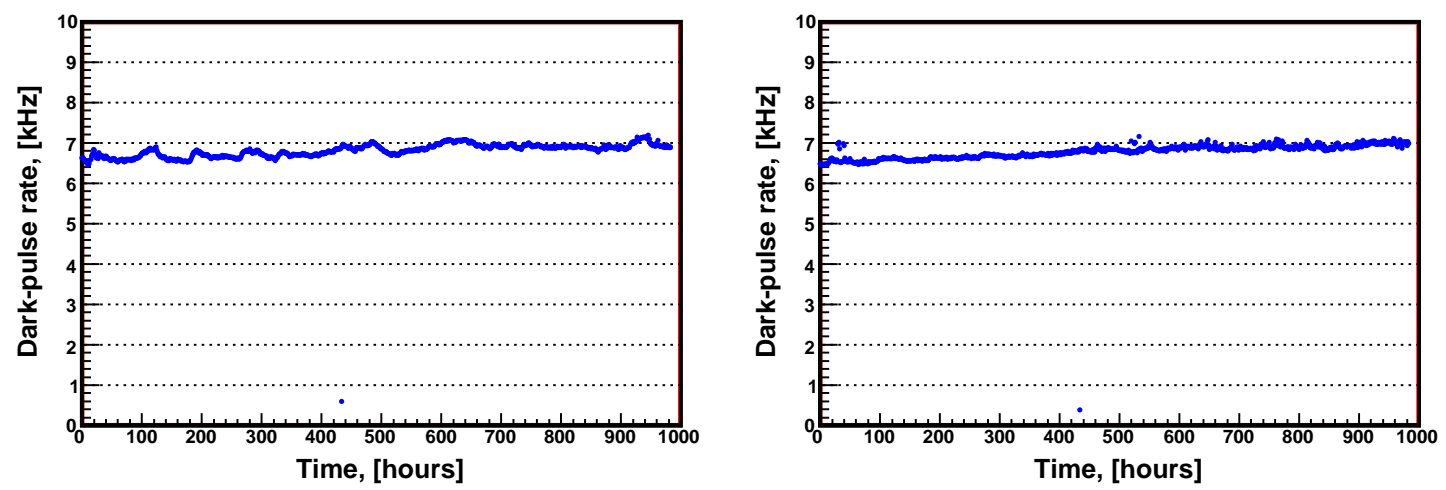

Figure 2: The dark-pulse rate at the discriminator level of 1/4 of p.e. of two 17-inch PMTs measured during the stability test in April-June of 1999.

ning of April to the end of June 1999. Typical ambient temperatures in April-May range between 10 and $15^{\circ} \mathrm{C}$. Toward the end of the test, temperatures had increased to $15-25^{\circ} \mathrm{C}$ which can be observed in the corresponding increasing trend of the dark-pulse rate owing to a higher thermionic emission from the photocathode for both PMTs. After the KamLAND detector was filled with LS and buffer oil, the dark-pulse rate of the ID PMTs turned out to be much higher than expected from the previous test measurements (Figure 2 ) and from the Kamiokande observations which showed that the dark-pulse rate of the majority of the 20-inch PMTs was less than $3 \mathrm{kHz}$ (at $10^{\circ} \mathrm{C}$ ) [5]. Figure 3 shows the dark-pulse rate of two of the KamLAND PMTs as a function of discriminator threshold value, operated under similar conditions (high voltage (HV), and temperature). Even before applying a correction for the photo-cathode area difference between the 17-inch and 20-inch PMTs, the dark-pulse rate (above the noise level) for the PMT located in ID (left plot) is about 10 times higher than that of the PMT in the OD (right plot). Both ID and OD PMTs are connected to identical HV power supplies and electronic readouts. Since the beginning of operations of the KamLAND detector, the PMTs HV power supplies were replaced several times but with
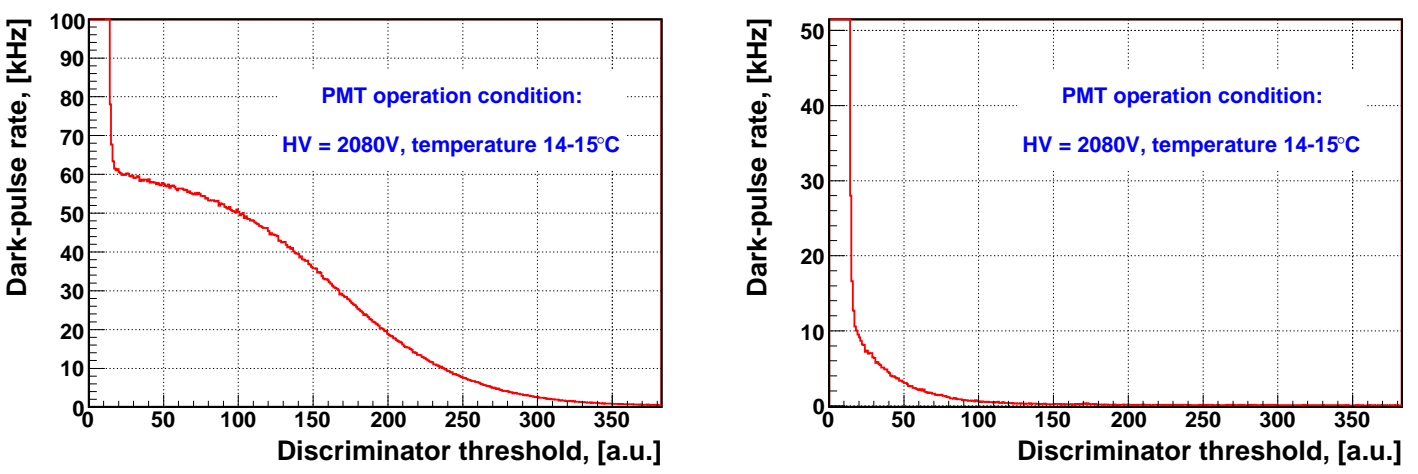

Figure 3: The dark-pulse rate of an ID 17-inch PMT (left) and an OD 20-inch PMT (right) as a function of discriminator threshold value measured in KamLAND after the beginning of a routine data collection. 

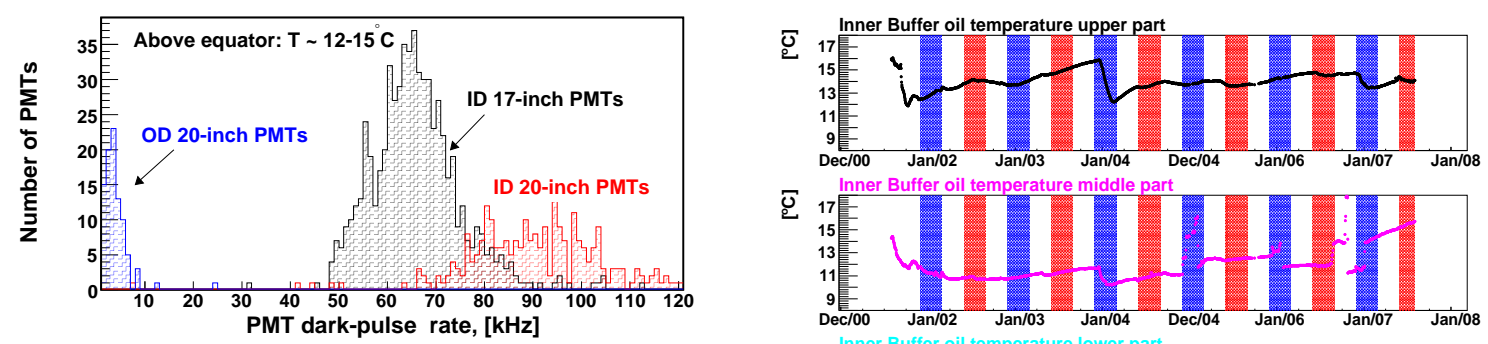

ర్ㄴ.
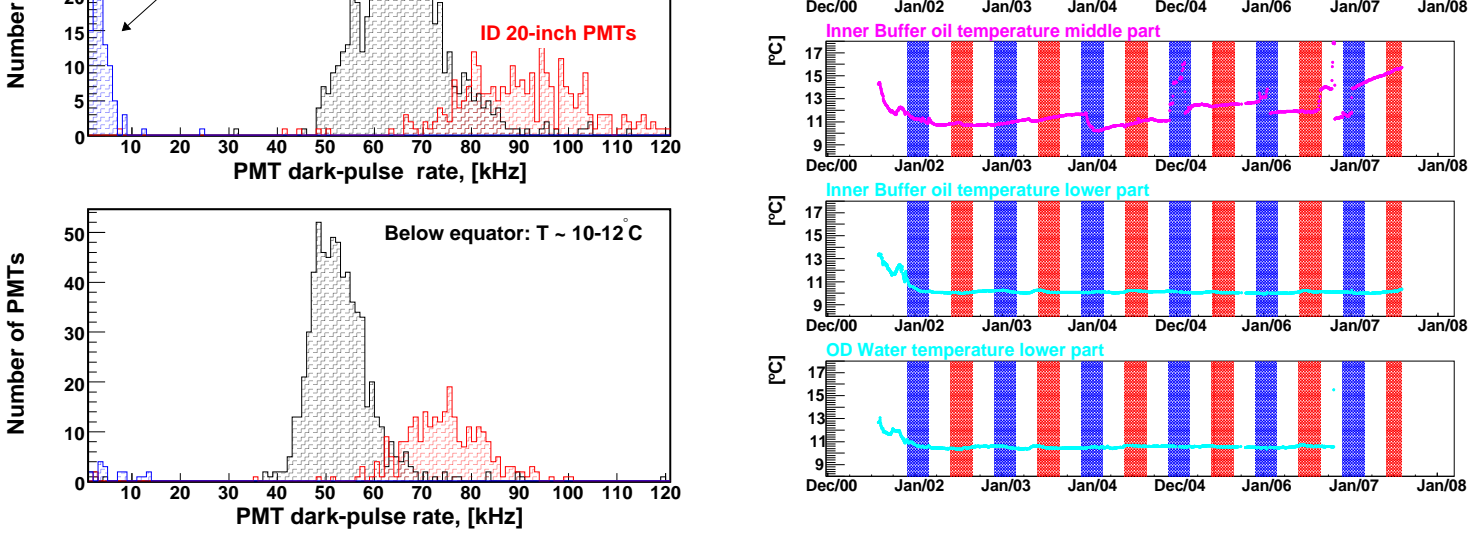

ভ্․

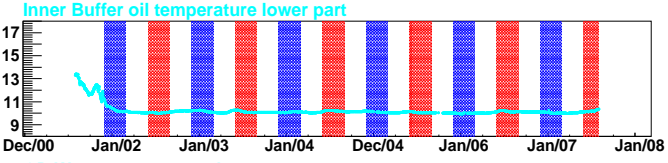

ত

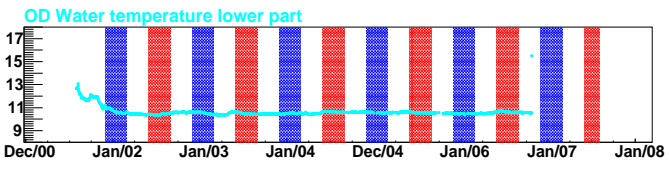

Figure 4: (Left) The dark-pulse rate of all the ID and OD PMTs at the discriminator threshold of $\sim 1 / 4$ of p.e.; (right) buffer oil and water temperature variations observed in different parts of the detector during 5 years of KamLAND operation.

no significant change in the dark-pulse rate above the noise level. $2 \mathrm{kWatt}$ of heat are released by all PMTs in KamLAND causing a stable vertical temperature gradient. The right plot in Figure $\emptyset$ shows buffer oil and OD water temperature variations in the lower, middle and upper parts of the detector during five years of its operation. The oil and water (as well as the LS) temperatures at the bottom have been relatively stable at $10^{\circ} \mathrm{C}$ while the water, oil, and LS temperatures at the top of the detector have fluctuated with the amount of pure water supplied to the OD. The dark-pulse rate values for all of KamLAND's 17-inch and 20-inch PMTs at the discriminator level of $\sim 1 / 4$ of p.e. observed in May 2003 are shown in Figure 4. Due to higher temperatures, the dark-pulse rate of PMTs located in the upper hemisphere of the ID is $20 \%$ higher than that of PMTs in the lower hemisphere. Typical dark-pulse rates of 20-inch OD PMTs range between 3 and $6 \mathrm{kHz}$, which is an order of magnitude lower than the dark-pulse rate of ID PMTs operated at the same temperature. The number of operational OD PMTs at the detector bottom is reduced compared to the top part due to faults in the design of their waterproof structure.

Figure 5 shows timing distributions of the 17 -inch PMTs signals for ${ }^{137} \mathrm{Cs}$ and ${ }^{203} \mathrm{Hg} \gamma$-ray events from calibration sources deployed in the center of the detector. The sharp peaks show the PMT signals from the calibration sources while the flat component results from the PMT dark pulses. A fraction of the dark pulses in the $150 \mathrm{~ns}$ timing window (see Figure 5 ) is $\sim 6 \%$ in ${ }^{137} \mathrm{Cs}$ and $\sim 15 \%$ in ${ }^{203} \mathrm{Hg}$. Moreover, at low energies the LS light output is no longer linearly proportional to ionization losses even for electrons. The light output 'quenching' described by the empirical Birks formula [6] causes an increase in relative contribution from the PMTs dark noise with a decrease of particle energy. In the KamLAND event reconstruction scheme, a sum of the PMTs signals with the charge above the 0.3 p.e. threshold level in the $150 \mathrm{~ns}$ timing window is used for the eventenergy calculation after subtracting the mean dark charge contribution. The mean dark charge value used for the correction is updated on a run-by-run basis to account for PMTs dark-pulse rate fluctuations which result from the detector temperature variations (see Figure 4 ). At low energies 

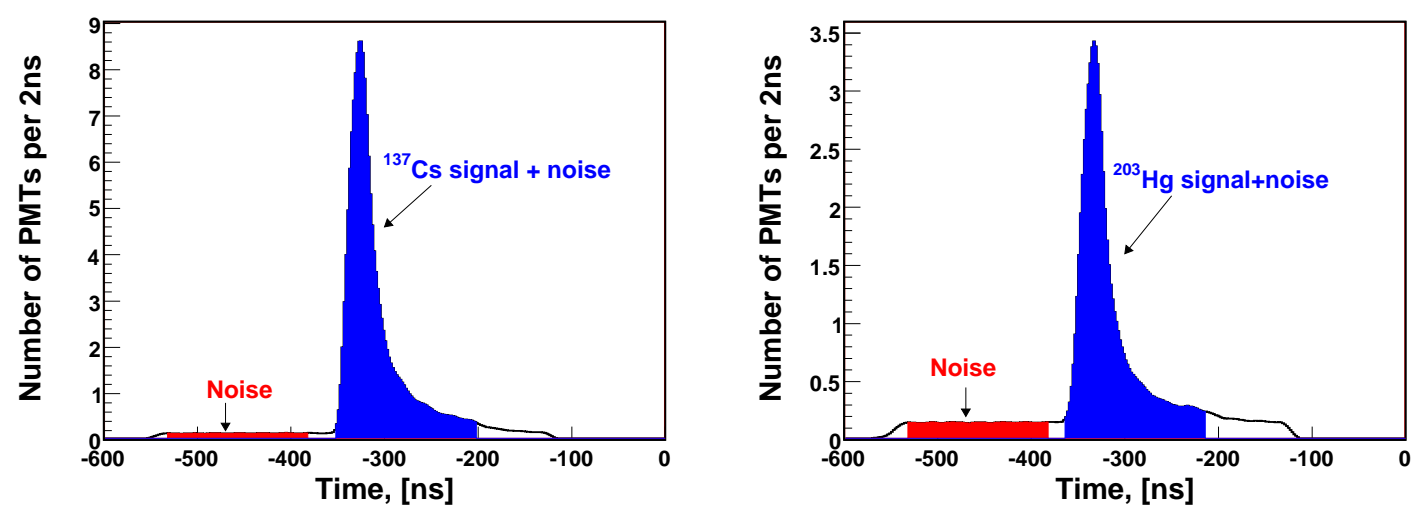

Figure 5: Timing distributions of the 17 -inch PMT signals obtained with the ${ }^{137} \mathrm{Cs} 661 \mathrm{keV} \gamma$-ray source (left); similar distribution for the ${ }^{203} \mathrm{Hg} 279 \mathrm{keV} \gamma$-ray source (right).

a relatively large fraction of an event's charge is due to the dark pulses. Therefore, a reduction of an energy-and-position reconstruction resolution is unavoidable.

$$
N_{P M T}=N_{\text {dark }}+N_{\text {rad }}+N_{\text {noise }}+N_{\text {light }}
$$

A PMT's total dark-pulse rate, $N_{P M T}$, defined by Eq. 1, results from several real and hypothetical components such as, $N_{\text {dark }}$, the dark-pulse rate component caused by thermionic emission from the photocathode, $N_{\text {rad }}$ signals produced by the scintillation and Cherenkov light from radioactive decays, $N_{\text {noise }}$, noise pulses, and, $N_{\text {light }}$, hypothetical PMT signals caused by light photons emitted by the KamLAND scintillator as the result of any chemical or physical processes not related to the light emission from radioactive decays. By comparing the OD and ID PMTs dark-pulse rate values, and by taking into account the laboratory and Kamiokande measurements, one can conclude that the dark-pulse rate component, owing to the thermionic emission from the photocathode of the KamLAND 17-inch or 20-inch PMT, is below $10 \mathrm{kHz}$. A $\sim 50 \mathrm{kHz}$ excess in the 17-inch PMT dark-pulse rate is equivalent to $50 \times 10^{3}$ p.e./s $\times 1325=6.6 \times 10^{7}$ p.e./s for all the 17 -inch KamLAND PMTs or $220 \mathrm{GeV} / \mathrm{s}$ of the energy deposition (assuming 300 p.e. per MeV [e]). For comparison, the energy deposition from cosmic-ray muons passing through the KamLAND ID is only $0.2 \mathrm{~Hz} \times 10^{6}$ p.e. $<1 \mathrm{GeV} / \mathrm{s}$. If the dark-pulse rate excess is a result of a light emitted by the LS, then the $6.6 \times 10^{7}$ p.e./s signal from all 17-inch PMTs corrected for the $22 \%$ photo-cathode coverage [2], and $\sim 20 \%$ photo-cathode quantum efficiency [5], gives a rough estimate for the number of photons emitted in the ID: $1.5 \times 10^{9}$ photons $/ \mathrm{s}$ per $1200 \mathrm{~m}^{3}$ or $1.25 \times 10^{3}$ photons/s per 1 litre of the LS. It is very unlikely that noise is a significant contributor to dark-pulse signals above the 0.3 p.e. level due to the following reasons. First, all of the KamLAND PMTs located both in the ID and OD are connected to the same electronic readouts and HV power supplies, while the OD PMT dark-pulse rates are consistent with the laboratory and Kamiokande measurements. Second, the ID PMT dark pulses have a mean charge of $\sim 1$ p.e. and are identical to normal PMT signals caused by light emitted by radioactive decay events. Third, a special measurement performed in the detector with only one ID PMT turned on recorded the same dark-pulse rate as during routine operation. 

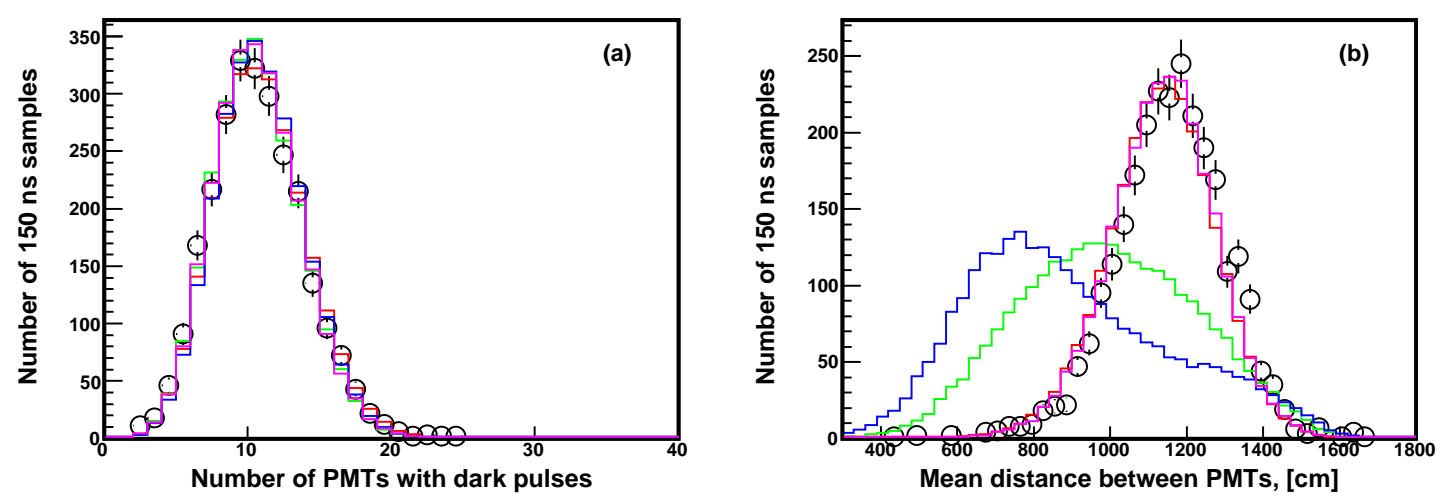

Figure 6: The KamLAND data points are shown by open circles. The Monte-Carlo simulation results are plotted as solid-line histograms: (a) number of the 17-inch PMTs with dark pulses detected in a $150 \mathrm{~ns}$ sampling interval; (b) the mean distance between the 17-inch PMTs with dark pulses detected in the $150 \mathrm{~ns}$ sampling interval.

The spatial distribution of the KamLAND PMTs with the dark pulses have been used to search for a dark-pulse rate component from very low energy radioactivity events. A low energy $\gamma$-ray is absorbed in a very compact region of the LS, while $\beta$-emitters, such as ${ }^{14} \mathrm{C}$, have a point-like vertex. Thus, the PMTs receiving photons from a compact source form a closely positioned cluster. A search for such PMT clusters was performed by comparing the mean distance between the KamLAND ID 17-inch PMTs with dark pulses (above 0.3 p.e. threshold) recorded within arbitrarily selected $150 \mathrm{~ns}$ intervals to results from Monte-Carlo simulations (MCS). A simple MCS program was used to generate photons in the LS volume and trace them to the known PMT locations. The MCS accounts for the real detector, PMT geometry and the light absorption in the buffer oil and the LS. The mean number of photons detected by PMTs in the MCS is the same as that observed in the KamLAND data (see Figure $6(a)$ ). Figure $5(b)$ shows distributions of the mean distances between 17-inch PMTs with dark pulses from the KamLAND data (open circles), and those generated by the MCS (solid line histograms). The blue histogram with the shortest distance between PMTs represents a MCS program run which generated all photons (per $150 \mathrm{~ns}$ sample) from a fixed vertex point while all vertexes were uniformly distributed on the KamLAND balloon surface. The green histogram shows the same MCS results with the only difference being that all vertexes were uniformly distributed within the KamLAND scintillator volume. These two MCS results simulate low energy radioactivity events originating from the balloon and the LS, respectively. Two other MCS histograms (red and magenta), identical to the real KamLAND data, were calculated using single photons emitted uniformly within the LS volume (without a single vertex), and for randomly selected PMTs. The last two MCS results show the hypothetical emissions of single light photons by the LS, and the thermionic emissions from the PMT photocathode respectively. A comparison between the data and the MCS indicates that the spatial distribution of the KamLAND PMTs with dark pulses cannot be explained by the presence of a radioactive contamination in the detector, unless their decay energy is less than $10 \mathrm{keV}$. In summary, the low energy radioactivity is not considered to be a substantial source of the KamLAND ID PMTs dark pulses which is shown 

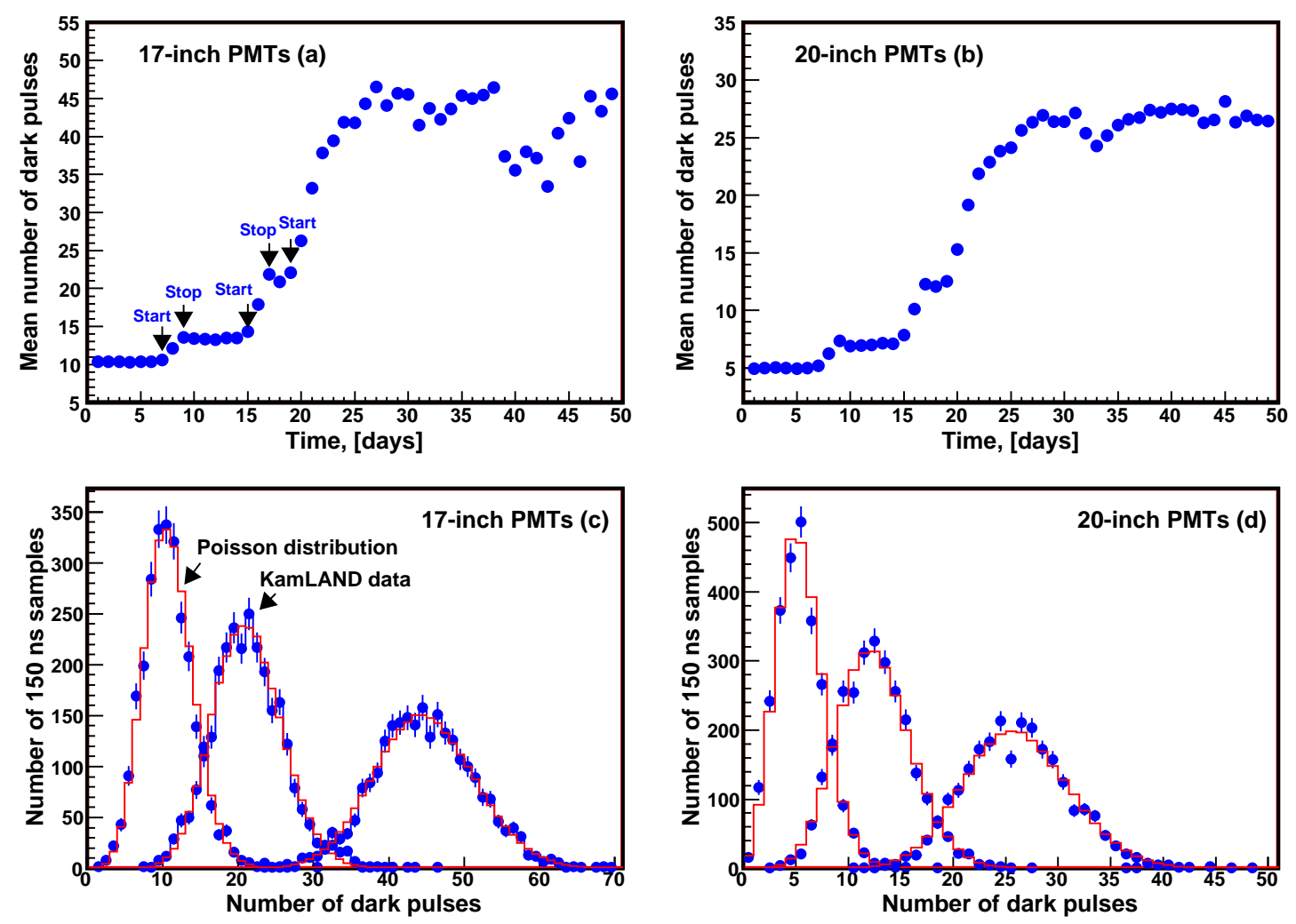

Figure 7: $(\mathrm{a}, \mathrm{b})$ Mean number of dark pulses above the 0.3 p.e. threshold from all 17 -inch or 20-inch ID PMTs in a $150 \mathrm{~ns}$ sampling window before and during the LS distillation; (c, d) examples of 17-inch and 20inch PMTs dark-pulse distributions used to calculate daily mean values shown in plots ( $a$ and $b$ ). The blue points with error bars are KamLAND data, and the red solid-line histograms are the Poisson distributions.

by the Poisson shape of the PMTs dark-pulse distribution (see Figure $5(\mathrm{a})$ ) and by the spatial distributions of the PMTs with dark pulses showing no sign of a compact vertex. The comparison between the MCS and experimental data, however, can not completely exclude the remote possibility of heavy contamination of the KamLAND LS with a low energy (a few keV) radioactive element which could produce only one PMT dark pulse in all the KamLAND PMTs. The signals from such very low energy background events are identical to the purely random dark pulses from the thermionic emission from the photocathode, or to single photons emitted uniformly by the LS. ${ }^{187} \mathrm{Re}$, a $\beta$-emitter with $\mathrm{E}_{\max }=2.7 \mathrm{keV}$, and $\mathrm{T}_{1 / 2}=4.35 \times 10^{10}$ years, can serve as an example of such a radioactive element. Fortunately, distillation procedures of the KamLAND LS, recently performed and described below, eliminate the possibility of such radioactive contamination as a possible explanation of the dark-pulse rate excess. Several other potential explanations such as slow 'phosphorescence' in the LS after exposure to a strong radioactive source $\left({ }^{60} \mathrm{Co}\right)$; a light emission due to chemical reactions caused by the scintillator's oxidation; or a light emission due to a static balloon tension were also tested but rejected based on results of small scale laboratory measurements.

The KamLAND's collaboration recent efforts to reduce the level of radioactive impurities 
in the KamLAND LS helped to understand the phenomenon of the high dark-pulse rate of the ID PMTs. During the purification process the LS was gradually removed from the bottom of the KamLAND balloon, transferred to the distillation facilities, and returned back to the balloon through a pipe line at the top of the detector. The LS flow rate was stable at 0.3 litre per second except for periods when the distillation process was suspended. Figure 7 shows the correlation between the number of dark pulses of the ID PMTs in a $150 \mathrm{~ns}$ window (only pulses above the 0.3 p.e. threshold are included) and the LS circulation status during a 50 day period before and after beginning of the distillation. In Figure $7(\mathrm{a}$ and $\mathrm{b})$ each point corresponds to the number of dark pulses from all 17-inch or 20-inch PMTs averaged over $\sim 2000$ of the 150 ns sampling intervals. Figure $\nabla(\mathrm{c}$ and, d) show three examples of the dark-pulse distributions for the 17-inch and 20-inch ID PMTs collected during different 24 hour periods where the blue points with error bars are the KamLAND data, and the red histograms are the Poisson distributions with the mean values given in Figure $7(\mathrm{a}$ and, b). One can see that the number of dark pulses detected within arbitrary $150 \mathrm{~ns}$ time intervals follows the Poisson statistics. Before the beginning of the distillation, the number of 17-inch (20-inch) PMTs dark pulses above the 0.3 p.e. threshold was stable at $\sim 10(\sim 5)$ pulses per 150 ns. In Figure 7 (a) the beginning or continuation of the LS circulation process is labelled as 'Start', and the time of a temporary interruption as 'Stop'. A clear correlation can be seen between the LS macroscopic motion caused by the LS distillation and the ID PMTs dark-pulse rate. The most likely explanation of this phenomenon is that the LS emits light photons during the macroscopic motion. Based on this observation the dark-pulse rate excess of the KamLAND ID PMTs can be explained by light emission from the LS which is the result of re-distribution of the $2 \mathrm{kWatt}$ of heat released by the PMTs which causes a slow motion of the LS and buffer oil. The final test requires switching all of the KamLAND PMTs off for a relatively long time period which cannot be done without causing a long dead time for the Super-Nova detection and, therefore not possible in the near future. The observed effect is likely to be proportional to the detector volume and may also depend on the scintillator chemical composition and operation conditions. Based on the results of our observations, we recommend that the R\&D phase of the next generation of large LS detectors, such as HANOHANO and LENA [7], must include studies of a possible negative influence of very high PMT dark-pulse rates on the detector energy-and-position reconstruction resolution.

I am grateful to Yuri Kamyshkov from The University of Tennessee, and Elena Kozlova from University of East Anglia for useful discussions and critical remarks.

\section{References}

[1] K. S. Hirata et al., Phys. Rev. D38, 448 (1988).

[2] K. Eguchi et al. (KamLAND Collaboration), Phys. Rev. Lett. 90, 021802 (2003).

[3] T. Araki et al. Phys. Rev. Lett. 94, 081801 (2005).

[4] H. Kume et al. Nucl. Instr. and Meth. 205, (1983) 443.

[5] A. Suzuki et al. Nucl. Instr. and Meth. A 329, (1993) 299

[6] J. B. Birks, Proc. Phys. Soc. A 64, 874 (1951).

[7] T. M. Undagoitia et al. Prog. in Part. and Nucl. Phys. 57, Issue 1, (2006) 283-289. 